\title{
FACTOR INDETERMINACY IN THE 1930'S AND THE 1970's SOME INTERESTING PARALLELS
}

\author{
JAMES H. STEIGER \\ UNIVERSITY OF BRITISH COLUMBIA
}

\begin{abstract}
The issue of factor indeterminacy, and its meaning and significance for factor analysis, has been the subject of considerable debate in recent years. Interestingly, the identical issue was discussed widely in the literature of the late 1920's and early 1930's, but this early discussion was somehow lost or forgotten during the development and popularization of multiple factor analysis. There are strong parallels between the arguments in the early literature, and those which have appeared in recent papers. Here I review the history of this early literature, briefly survey the more recent work, and discuss these parallels where they are especially illuminating.
\end{abstract}

Key words: factor analysis, factor indeterminacy.

Since 1970, a number of articles have discussed the issue of "factor indeterminacy," and its meaning and significance for common factor analysis. The discussions have often been both esoteric and polemical, and the practical psychometrician may find the meaning of it all somewhat obscured. Although factor indeterminacy may appear to be a relatively new issue, it actually has a long and interesting history, dating back to the early 1920's. This early literature was either ignored or forgotten during the growth and popularization of multiple factor analysis in the 1930's and 1940's, to the extent that major textbooks of the period [e.g., Thurstone, 1935; Holzinger \& Harman, 1941; Thurstone, 1947] never even mentioned the existence of factor indeterminacy. The modern reader, trying to form his own opinion about indeterminacy, will find some of the points made in these early papers well worth remembering. The papers are interesting in their own right, and there are some interesting parallels between the issues which were discussed in the 1930's, and those which have emerged in the 1970's.

The main purpose of the present account is to define factor indeterminacy and the related issues in relatively non-technical terms, and to review the entire history of factor indeterminacy, drawing parallels between old and new work where such parallels are especially illuminating.

There are, of course, strong differences of opinion concerning the importance of factor indeterminacy for factor analysis. My opinion [see, e.g., Steiger, 1979; Schönemann \& Steiger, 1976] is that indeterminacy and related problems of the factor model counterbalance the model's theoretical advantages, and that the elevated status of the common factor model (relative to, say, component analysis) is largely undeserved. Other writers disagree very strongly with this view. The purpose here is to present a historical review while avoiding strong criticisms of either position. Consequently, readers with strongly polarized views on factor indeterminacy may well find this treatment rather bland and uncritical. Hopefully they will agree that it is unbiased and relatively complete.

Support by the NRC (Grant No. A4640) and the University of British Columbia (UBC Humanities and Social Sciences Grant 26-9508) is gratefully acknowledged.

Requests for reprints should be sent to James H. Steiger, Department of Psychology, University of British Columbia, Vancouver, B.C., CANADA V6T IW5. 


\section{Factor A nalysis and Factor Indeterminacy-Some Basic Theory}

Given some $p$ observed random variables in the random vector $y$, with $\delta(y)=\phi$, $\operatorname{var}(y)=\varepsilon\left(y y^{\prime}\right)=C_{y y}$, the $m$-factor orthogonal common factor model holds in the population if one can write

$$
y=A x+U z
$$

where $x$ is an $m \times 1$ random vector of $m$ "common factors," $z$ is a $p \times 1$ random vector of $p$ "unique factors," $A$ is a $p \times m$ matrix of constants, of full column rank, called the "common factor pattern," and $U$ is a $p \times p$, diagonal, positive definite matrix of coefficients called the "unique factor pattern," with $x$ and $z$ satisfying the conditions

$$
\mathcal{E}\left(x x^{\prime}\right)=I, \mathcal{E}\left(x z^{\prime}\right)=\phi, \mathcal{E}\left(z z^{\prime}\right)=I, \mathcal{E}(x)=\phi \text {, and } \varepsilon(z)=\phi .
$$

It is well-known that (1) and (2) hold for $y$ if and only if one can write

$$
C_{y y}=A A^{\prime}+U^{2} \text {. }
$$

Equation (3) is often called the "fundamental theorem of factor analysis."

If a $U^{2}$ is uniquely available to reduce $C_{y y}$ to rank $m$, then $A$ is uniquely defined up to a rotation. For any rotated $A$, however, there exist infinitely many different $x, z$ which satisfy (1) and (2). Specifically, Guttman [1955] has shown that, if $s$ is any $m \times 1$ random vector satisfying

$$
\mathcal{E}\left(y s^{\prime}\right)=\phi, \quad \mathcal{E}\left(s s^{\prime}\right)=I, \quad \text { and } \quad \mathcal{E}(s)=\phi,
$$

then any and all $z, x$ satisfying (1) and (2) can be constructed as

$$
x=A^{\prime} C_{y y}^{-1} y+P s \quad \text { and } \quad z=U C_{y y}^{-1} y-U^{-1} A P s,
$$

where $P$ is any Gram-factor of $I-A^{\prime} C_{y}^{-1} A$.

Equation (5) can be rewritten

$$
x=B^{\prime} y+e=\hat{x}+e .
$$

In this form, it can easily be seen that $x$, the random vector of common factors, is the sum of two components. One, $\hat{x}$, is a determinate linear combination of the observed variables. The other, $e$, is an arbitrary random vector orthogonal to $\hat{x}$. Using the arbitrary $e$, we can construct infinitely many different $x$ 's for any given $y, A, U$. Some of these $x$ 's will be quite different.

Although factor indeterminacy has been presented in terms of population random vectors, it should be noted in passing that the identical situation holds in the sample. Factor indeterminacy is thus a structural aspect of the common factor model, stemming from the fact that the model has more latent variables than observed variables. It is not a sampling problem which can be alleviated by adding more subjects to a study, for example.

\section{Factor Analysis from 1900 to 1920}

Charles Spearman proposed his single common factor model, the "theory of $g$," in 1904 , as a mathematical, empirically falsifiable psychological model. He and his associates then launched a vigorous program of experimental verification, culminating almost a decade later in the classic article by $\mathrm{Hart}$ and Spearman [1912]. Hart and Spearman portrayed factor analysis as the central focus of a new educational technology, from which would ensue a wide range of practical benefits. They wrote enthusiastically of a veritable psychometric utopia, in which every citizen would have his "intellective index" measured and registered with the government, and decisions about vocations, voting rights, and even the right to have offspring would be made on the basis of this index. If Spearman 
seemed overenthusiastic, he had just cause. The data, in overwhelming quantity, seemed to provide strong support for his notion of a single general intelligence factor. As S. C. Dodd [1928a] noted in a historical review some years later, "It seemed to be the most striking quantitative fact in the history of psychology." (p. 214)

Godfrey H. Thomson's sampling theory of abilities provided Spearman's theory with its most serious challenge during this early period. Thomson's theory asserts that the mind is composed of an extremely large number of components, and that some of these (higher level units) participate in many different kinds of activities, while others (lower level units) are restricted to a single kind of activity. Any given task is performed using a random sample from the appropriate units of both levels. Thomson [1916, 1919] accepted Spearman's data, but insisted that his model fit the data as well as Spearman's.

During this period, one focus of debate was whether the tetrad difference criterion was sufficient to prove the actual "existence" of $g$, Spearman's general ability factor. Aiming to resolve the controversy, Spearman concentrated on showing a necessary compatibility between hierarchical correlational structure, and a fit to a single common factor model.

Along these lines, Garnett [1919] gave a proof that, in the case of normally distributed variables, a correlation matrix exhibiting hierarchical structure must be compatible with the Spearman model. Comparing Spearman's theory with Thomson's, Garnett [1920] expressed what was probably the prevailing view at the time, i.e., that the former was preferable for its parsimony.

\section{Factor Indeterminacy from 1922 to 1930}

By 1922, Spearman had firmed up the algebra of his model considerably, and he gave a proof [Spearman, 1922] that, if observed correlations satisfy a tetrad difference criterion, scores can be constructed which satisfy perfectly the definition of " $g$-factor scores." Spearman provided a determinantal expression, which can be shown to be the singlefactor sample equivalent of (4)-(6), for calculating these scores.

The proof was repeated, almost verbatim, 5 years later, in the mathematical appendix to The Abilities of Man [Spearman, 1927]. Spearman's view at the time was that his mathematics (together with an erroneous proof in Garnett, 1919, that $g$ was uniquely defined) and his data provided proof of the existence of a uniquely defined general intelligence factor. Emphasizing the significance of Garnett's proof, Spearman wrote:

There is another particularly important limitation to the divisibility of the variables into factors. It is that the division into general and specific factors all mutually independent can be effected in one way only; in other words, it is unique. For the proof of this momentous theorem, we have to thank Garnett. [Spearman, 1927, p. vii]

The following year, reviewing The Abilities of Man for Science, E. B. Wilson, a leading mathematician of that period, pointed out that different sets of factor scores could fit Spearman's model perfectly for the same set of data. Wilson's treatment [1928a] was noteworthy, because it provided a simple numerical example of the effect of indeterminacy, and also attempted to characterize this effect numerically. Wilson derived the intelligence factor scores for 6 students on 3 tests, and showed that each of these scores was free to vary over a wide range. Wilson saw this wide range of possible definition as creating a real dilemma for Spearman and others who would attempt to make practical use of intelligence factor scores. What good were such scores if one could alternatively assign a student a very low or a very high intelligence score? Under such conditions, would it be proper to view $g$ as uniquely defined?

In a later paper the same year, Wilson [1928b] gave a geometric proof that $g$ could be 
expressed as the sum of two components, one a determinate linear combination of the original variables, the other an indeterminate largely arbitrary component. He thus confirmed what was already inherent in Spearman's [1922, 1927] algebra, but gave his results a much different interpretation.

Following a third Wilson [1929a] article on factor indeterminacy, Spearman [1929] published a brief article in defense of his model. While Wilson portrayed factor indeterminacy as a lack of uniqueness in the variable $g$ itself, Spearman presented the issue in a different light. He suggested that the indeterminacy of $g$ was easily eliminated by adding $a$, a test constructed to correlate perfectly with $g$, to the test battery. Spearman indicated that "unpublished research in our laboratory has more than once obtained for an $r_{a g}$ values of .99 ," adding that "nothing stands essentially in the way of raising it much higher still; in fact as near as desired to unity." [Spearman, 1929, p. 214]

In a brief rejoinder, Wilson [1929b] pointed out that the existence of such an $a$ would imply that the test was perfectly reliable, and that if such a test were to exist, we could "throw away our scaffolding," i.e., forget about factor analysis entirely, and simply make test $a$ the measure of $g$. Wilson might have added that, since there was more than one $g$, there would obviously be more than one $a$, and so adding a particular $a$ would be tantamount to selecting (arbitrarily) a particular $g$.

\section{Factor Indeterminacy from 1931 to 1939}

The period from 1931 to 1939 was one of intense development and controversy in factor analysis in general, and factor indeterminacy in particular. We will begin by describing the technical and theoretical developments, and then give details of major areas of conflict.

\section{Technical Developments}

A number of writers in the 1930's added to the early foundation work in factor indeterminacy. Their technical and theoretical developments can be summarized under three major themes:

The construction approach. Piaggio [1931] simplified Spearman's [1922] determinantal formula for $g$, and showed explicitly that $g$ could be divided into a determinate and an indeterminate part. Irwin [1932] pointed out the close relationship between the mathematics of Wilson [1928b] and Piaggio [1931]. In 1933, Piaggio gave a much more explicit statement and proof of his 1931 result, which had since been verified by Heywood's [1931] independent derivation. Piaggio showed that $g$ can be expressed as in (4)-(6), and gave details of how different sets of common factor scores could be constructed by arbitrarily varying the vector $s$. This approach to indeterminacy later became known as the "construction approach," because a method for constructing any and all sets of factor scores fitting the common factor model is derived simultaneously with the demonstration of indeterminacy.

Piaggio [1933] demonstrated the sufficiency of (4)-(5), i.e., that any set of numbers constructed by the sample equivalent of these equations would fit the factor model in the sample equivalents of (1)-(2). Furthermore, in 1935, Piaggio demonstrated the necessity of (4)-(6), thus establishing that any and all sets of factor scores satisfying the factor model must be expressible in terms of the sample equivalents of the construction equations.

Indeterminacy in the limit. If the single common factor model continues to hold as more and more tests are added to the test battery, then the variance of the indeterminate part of $g$ becomes increasingly small. This indeterminate part vanishes, in fact, as the number of tests becomes infinitely large. This was first pointed out by Spearman [1922], amplified in Piaggio [1931], and clarified in Piaggio and Dallas [1934], and Irwin [1935]. 
Thus, if a single common factor can fit the factor model for an infinite number of variables, this factor will be uniquely defined.

The transformation approach. Thomson [1935] introduced an approach to indeterminacy which Schönemann and Wang [1972] later called the "transformation approach." Suppose the factor model, for a given $A, U$, is written in the form

$$
y=\left[\begin{array}{lll}
A: U & U
\end{array}\right]\left[\frac{x}{z}\right] .
$$

Thomson [1935] gave a formula for a transformation matrix $B$ with the properties

$$
B B^{\prime}=I, \text { and }[A: U] B=[A: U] .
$$

The existence of a $B$ satisfying (8) implies factor indeterminacy, since one may then write

$$
y=[A: U] B B^{\prime}\left[\frac{x}{z}\right]=[A: U]\left[\frac{x^{*}}{z^{*}}\right] \text {. }
$$

Thus, for any factors $x, z$, satisfying the factor model with $A, U, y$, there exist different factors, $x^{*}, z^{*}$ which also satisfy the model. Ledermann [1938] extended Thomson's result to the multiple factor case.

\section{Areas of Controversy}

The technical facts of indeterminacy were established with little debate, but there were major differences of opinion over the interpretation of these facts, which are summarized briefly below.

Indeterminacy-lack of uniqueness or error of measurement? Wilson characterized factor indeterminacy as a lack of uniqueness of $g$, the Spearman model's central concept. Camp [1932, 1934] shared this view, and forcefully expressed the idea that this lack of uniqueness seriously compromised the practical value of the Spearman model:

If, before looking at Smith's scores on the tests, one may choose a number at random (subject only to the broad limitations mentioned before), and can then demonstrate that this number can be assigned as Smith's $g$, as well as any other number, and in perfect harmony with all the other hypotheses, then it is meaningless to assert that Smith has a $g$. [Camp, 1934, p. 261]

Spearman, however, had a different view of the situation. He conceptualized $g$, the general intelligence factor, as a quality of mind which must in principle be defined uniquely. The apparent indeterminacy of $g$ was for Spearman merely an aspect of the mathematics of factor analysis. Citing the similarity between (6) and the algebra of multiple regression, Spearman [1933, 1934] characterized factor indeterminacy as essentially a sampling problem, nothing more than an error of regression estimate. This error, according to Spearman, could be reduced as much as desired by simply adding more tests to the test battery. (Although it may not be immediately obvious, this argument was very similar to his earlier [1929] position. Instead of adding one test to improve the indeterminacy of $g$, Spearman now proposed to add many.)

Thomson [1934] attempted a rebuttal of Spearman's "regression approach" argument by pointing out some distinctions between factor construction and multiple regression. In multiple regression, the criterion is defined (and can in principle be measured) independently of the predictors. It is thus determinate. In factor analysis, the factor $g$ is defined implicitly as a latent variable which is never directly measureable. The indeterminacy of $g$ stems from the fact that it is defineable only in terms of its ability to satisfy (4)-(6).

In regression analysis, the additional variables which improve prediction can be any 
variables not linearly redundant with the original variables. In factor analysis, the variables must satisfy highly restrictive constraints in order not to disconfirm the hypothesis of a single common factor.

The basic conflict between the "regression" and "lack of uniqueness" positions did not re-emerge in the literature of the 1930's. However, some 40 years later it has resurfaced to form a major focus for the ongoing factor indeterminacy controversy.

The measurement of indeterminacy. Advocates of the lack of uniqueness position stressed the conceptual difficulties inherent in the existence of disparate, conflicting solutions for $g$. The greater the possible conflict in empirically indistinguishable factor scores, the greater the indeterminacy problem, since it was difficult to conceive of a meaningful concept of $g$ which could alternatively assign a person vastly different scores. In keeping with this view, Wilson [1928a] and Camp [1932] quite naturally used the range of possible solutions as an index of the severity of indeterminacy.

Spearman and Piaggio, proponents of the regression view, characterized indeterminacy as linear unpredictability of the factor $g$ from the original test variables. They used variants of the multiple correlation coefficient between $g$ and the original tests as numerical indices of indeterminacy. Along these lines, Piaggio [1933] offered two indices. One is the ratio of the standard deviations of the indeterminate and determinate parts of $g$. The second, the square of the first, is the ratio of indeterminate to determinate variances. Spearman [1933, p. 108] expressed a strong preference for the latter index, primarily because it always yields smaller numbers.

Thus, methods for numerically characterizing the extent of indeterminacy were intimately related to the theoretical conception of indeterminacy itself. This trend has persisted into the 1970's.

\section{The Thurstonian Era: 1940-1951}

Although factor indeterminacy was a key theoretical issue in the 1930's, it was all but ignored in the following decade. This attitude of indifference was foreshadowed by a historical review [Wolfle, 1940] which, although it purported to be a survey of important developments, including "limitations of factor analysis," never directly mentioned the existence of factor indeterminacy.

During the 1940's, Thurstone and his associates popularized "multiple factor analysis," an extension (originally proposed by Garnett in 1919) of Spearman's model. The decade witnessed a number of statistical and computational developments of major significance, including the introduction and widespread acceptance of the simple structure concept as a solution to the rotation problem, and Lawley's pioneering work in maximum likelihood factor analysis.

The computational and statistical advances of the 1940's were impressive, especially when one considers the disruptive influence of World War II. Unfortunately, factor indeterminacy and a related problem which Wilson had uncovered (the "unidentifiability of $U^{2}$ ") were either forgotten or ignored, to the extent that even the major texts [e.g., Holzinger \& Harman, 1941; Thurstone, 1947] failed to mention their existence.

\section{The Era of Blind Factor Analysis: 1952-1969}

The computational and statistical developments of the 1940's accelerated in the ensuing 2 decades, as factor analytic methodology became increasingly refined and computer technology rendered its application virtually effortless. As factor analysis became available to a broad spectrum of users (many of whom were theoretically unsophisticated), it was often applied uncritically, as a popular data reduction technique for rescuing poorly conceived correlational studies. Reviewing the history of the era, Mulaik characterized it as "the era of blind factor analysis." [Mulaik, 1972, p. 9]. 
Factor indeterminacy was largely ignored during this period, although there were some major papers produced. Kestelman [1952] extended some of the earlier work of Piaggio to the multiple factor case by showing that if $C_{y y}, A, U$ satisfy (3), then a matrix of factor scores fitting the common factor model can always be constructed.

Guttman [1955] contributed an extensive paper which provided a number of important theoretical developments:

(i) He derived construction formulae for orthogonal and oblique factors in both sample and population cases, and demonstrated the necessity and sufficiency of the construction formulae.

(ii) $\mathrm{He}$ introduced the minimum correlation between alternative factors as a numerical index of indeterminacy. Guttman examined the relation between this correlation, which he called $\rho^{*}$, and $\rho$, the multiple correlation between the factor and the observed variables. The relation is

$$
\rho^{*}=2 \rho^{2}-1 \text {. }
$$

Discussing the fact that $\rho^{*}$ could often be negative, Guttman concluded that factor indeterminacy posed a serious problem for factor theorists who considered a factor to be identified by a set of factor loadings.

... it seems that the sought-for traits are not very distinguishable from radically different possible alternative traits for the identical factor loadings. [Guttman, 1955, p. 74]

(iii) He questioned the usefulness of the concept of second order factoring, since such factors would generally be highly indeterminate.

(iv) He greatly extended earlier work on indeterminacy in the limit, by establishing conditions under which indeterminacy vanishes in the limit in the multiple factor and population cases.

Guttman contended that the common practice of naming factors according to the variables that have high loadings on them was illogical, when for any set of loadings, there exist an infinite number of different factor solutions. He concluded that "the SpearmanThurstone approach may have to be discarded for lack of determinacy of its factor scores." (p. 79)

Guttman's negative conclusions failed to strike a responsive chord within the American psychometric community. There were no new theoretical developments in factor indeterminacy in the 1960's, although Heermann $[1964,1966]$ summarized many of the known facts in two very readable reviews.

\section{Factor Indeterminacy from 1970 to 1977}

After 3 decades of relative inactivity, factor indeterminacy has again become the subject of debate. More articles on the subject have appeared since 1970 than in the previous 30 years combined. Although indeterminacy has hardly become a "popular" issue, sections on the problem in recent texts [Mulaik, 1972; Gorsuch, 1974] appear to reflect an increased awareness of its existence.

Schönemann [1971] derived a simplified formula for the minimum average correlation between equivalent sets or (orthogonal) common and unique factors, given by

$$
\bar{r}_{\mathrm{min}}=\frac{p-m}{p+m}=\frac{1-\frac{m}{p}}{1+\frac{\bar{m}}{p}},
$$

where $p$ is the number of original variables, and $m$ is the number of orthogonal common factors. 
Meyer [1973], using results from Guttman [1955, 1956], derived an equation relating the average indeterminacy of both common and unique factors to the ratio of the number of factors to number of variables. results:

Schönemann and Wang [1972] derived a number of new theoretical and empirical

(i) If $A$ and $U$ are identified by the diagonality constraint

$$
A^{\prime} U^{-2} A=\text { diagonal }
$$

then the uncorrelated common factors associated with $A$ are ordered from most to least determinate among all common factors obtainable by orthogonal or oblique rotation.

(ii) The numerical measure of indeterminacy for these factors is a simple function of the latent roots of an eigenproblem which is routinely solved in the course of maximum likelihood factor analysis (MLFA). Thus, the factor analyst using MLFA can simultaneously assess goodness of fit and factor indeterminacy in an easy and convenient way.

(iii) Factor scores fitting the factor model can be computed from the maximum likelihood estimates of $A, U^{2}$, and $Y$, whether $C_{y y}$ fits the factor model or not.

(iv) Data from 13 different factor analytic studies were reanalyzed to assess the extent of the factor indeterminacy which the factor analyst could expect to encounter in practice. Some major findings emerged: first, as the number of variables in a study increases, the number of factors required to achieve an adequate statistical fit also increases. On the other hand, increasing the number of factors generally leads to highly indeterminate common factors. Finally, an additional problem, the emergence of "doublets" (indicative of an unidentifiable factor pattern), also occurred when the number of common factors was increased. The results suggest that the factor analyst would often be caught in a dilemma: either have indeterminate factors or unidentifiable patterns, or a model that fails to fit the data adequately.

In conclusion, Schönemann and Wang challenged the logical foundations of the various factor score estimation procedures [Harris, 1967; McDonald \& Burr, 1967], which estimate a subject's factor scores with various linear functions of the observed variables. Early advocates of such procedures [e.g., Piaggio, 1935] apparently felt that only one of the available sets of scores which could be constructed via the sample analogs of (4)-(6) was the "true" set. Hence, arbitrary construction of a set of scores via the construction formulae would lead to factor scores which would fit the factor model, but which would not be "correct." Various estimation procedures were proposed partly as a compromise among the various alternative factor score solutions.

Although factor score estimation originated as a compensatory response to factor indeterminacy, this fact tended to become obscured in many later discussions. (For example, Harman's, 1967, text is strikingly opaque in this area-it devotes an entire chapter to factor score estimation procedures without ever discussing any of the significant work on factor construction or factor indeterminacy.) One statement which often (in minor variations) invaded discussions of factor score estimation was that "factor scores cannot be computed-they must be estimated."

Schönemann and Wang contended that this statement was at best misleading, because factor scores fitting the common factor model could indeed be computed. Factor scores cannot be computed uniquely, but this state of affairs should be distinguished from a lack of computability.

Factor score estimation was originally justified [Piaggio, 1935] as a solution to an indeterminacy problem which was seen as far from trivial. Indeed, if factor scores (which are computable) were uniquely computable, we would obviously have no need of factor score estimation. Nevertheless, by the early 1970's, many factor analysis texts failed to discuss the foundations of the indeterminacy problem and its intimate relation to factor 
score "estimation." Schönemann and Wang did not advocate using one of the computable sets of factor scores in place of factor score estimates. Rather, they saw factor score indeterminacy and factor unidentifiability as major problems for factor analysis, problems which, if not obscured by misleading terminology, might motivate more serious consideration of alternative data reduction methods, such as component analysis.

McDonald [1974] strongly opposed the conclusions of Schönemann and Wang, and based this opposition on two major arguments. First, he contended that "common factors are not subject to indeterminacy to the extent that has been claimed, because the measure of indeterminacy that has been adopted is ill-founded." (p. 203) McDonald argued that the minimum correlation index advocated by Guttman as a measure of indeterminacy was logically inconsistent, because its use implied that different values of a random variable could be associated with a subject at the same time. He pointed out that indeterminacy seems much less severe when measures such as the multiple correlation between the factor and the observed variables are used.

McDonald's second major point involved the supposition that one of the sets of factor scores fitting the observed data was the "true" set. Under these conditions, McDonald demonstrated that a factor analyst who uses regression estimates will almost inevitably be closer to the "true" factor scores than one who uses an arbitrarily constructed set of factor scores obtained from (4)-(6).

There is an interesting parallel between the discussion of measures of indeterminacy in McDonald [1974], and that in Spearman [1933], as both writers saw the measure of indeterminacy as affecting our conception of its importance, and both argued against the use of an overly pessimistic measure.

Mulaik [1976], de-emphasized the significance of this distinction, concluding that "it matters little" whether we use $\rho^{2}$ or $2 \rho^{2}-1$, "if we keep in mind that these 2 indices measure different aspects of the same situation." Mulaik proved that, when alternative solutions for a factor are equally likely to be chosen, then the squared multiple correlation $\rho^{2}$ for predicting the factor from the observed variables is the average correlation $\rho_{A B}$ between independently selected alternative solutions $A$ and $B$.

Green [1976] gave a brief review of the more recent factor indeterminacy literature, and offered a resolution to what he termed the "factor score controversy." He began by pointing out the correspondence between the equations of multiple regression and factor construction, and his discussion has interesting parallels to the earlier work by Spearman [1933] and Thomson [1934]. Green rejected McDonald's [1974] contention that one of the available sets of factor scores need be conceived as the "true" set. Instead, he justified the use of regression estimates on the grounds that they estimated all of the available sets of factor scores equally well. In conclusion, he noted that "It is unnecessary to assume that one of the infinite sets of admissable factor scores is true; they are all equally true, as all are estimated equally well by the factor score estimates." (p. 265)

\section{Conclusion}

As I mentioned at the outset of this brief historical review, there are strong differences of opinion about the meaning and significance of factor indeterminacy for factor analysts. There are of course many nuances which depend on one's conceptualization of the role of factor analysis itself, but the major theoretical positions tend to cluster around the "lack of uniqueness" and "regression" views.

Proponents (myself included) of the lack of uniqueness view stress the fact that factor analysis (even if it could be performed on population correlation matrices) does not uniquely identify its factors-rather, it identifies a range of random variables which can all be considered factors. This lack of uniqueness raises some interesting dilemmas for those who consider factors to have been "identified" by a set of factor loadings. 
Proponents of the regression position stress the values of factor analytic theory as opposed to more modest systems like component analysis. In the "regression" view, factors underlying a domain of variables can be reasonably well-identified, and factor indeterminacy should be viewed as a lack of precision which stems from sampling a limited number of variables from the domain of interest.

Although no clear resolution of the indeterminacy controversy seems imminent, some very recent research gives promise of further clarification of basic issues [Schönemann \& Steiger, 1978; Steiger, 1979; Mulaik \& McDonald, 1978; Williams, 1978]. These papers provide some interesting guidelines for future research trends in the area.

Regardless of how and when the factor indeterminacy controversy is resolved, it seems fair to say that the revival of interest in the 1970's has had a number of positive side effects for psychometrics. First, knowledge of the common factor model itself has been expanded considerably by the scrutiny of those on both sides of the controversy. Second, history has reemphasized the important lesson that it is counterproductive to dism:ss important theoretical issues (to the extent of forgetting that they ever existed), simply because counterarguments are available. Finally, we have corrected a mistaken impression, prevalent among our psychological colleagues, that psychometrics is a dry, uninteresting science, devoid of conflict, excitement, or controversy.

\section{REFERENCES}

Camp, B. H. The converse of Spearman's two-factor theorem. Biometrika, 1932, 24, 418-427.

Camp, B. H. Spearman's general factor again. Biometrika, 1934, 26, 260-261.

Dodd, S. C. The theory of factors, I. Psychological Review, 1928a, 35, 211-234.

Dodd, S. C. The theory of factors, II. Psychological Review, 1928b, 35, 261-279.

Garnett, J. C. General ability, cleverness, and purpose. British Journal of Psychology, 1919, 9, 345-366.

Garnett, J. C. The single general factor in dissimilar mental measurements. British Journal of Psychology, 1920 , 10, 242-258.

Gorsuch, R. L. Factor analysis. Philadelphia: Saunders, 1974.

Green, B. F. On the factor score controversy. Psychometrika, 1976, 41, 263-266.

Guttman, L. The determinacy of factor score matrices with implications for five other basic problems of common factor theory. British Journal of Statistical Psychology, 1955, 8, 65-81.

Guttman, L. "Best possible" systematic estimates of communalities. Psychometrika, 1956, 21, 273-285.

Harman, H. H. Modern factor analysis (2 ${ }^{\text {nd }}$ ed.). Chicago: University of Chicago Press, 1967.

Harris, C. W. On factors and factor scores. Psychometrika, 1967, 32, 363-379.

Hart, B., \& Spearman, C. General ability, its existence and nature. British Journal of Psychology, 1912, 5. 51-84.

Heermann, E. F. The geometry of factorial indeterminancy. Psychometrika, 1964, 29, 371-381.

Heermann, E. F. The algebra of factorial indeterminancy. Psychometrika, 1966, 31, 539-543.

Heywood, H. B. On finite sequences of real numbers. Proceedings of the Royal Society of London, 1931, 134, 486501.

Holzinger, K. J., \& Harman, H. H. Factor analysis. Chicago: University of Chicago Press, 1941.

Irwin, J. O. On the uniqueness of the factor $g$ for general intelligence. British Journal of Psychology, 1932, 22, 359-363.

Irwin, J. O. On the indeterminacy in the estimate of $g$. British Journal of Psychology, 1935, 25, 393-394.

Kestelman, H. The fundamental equation of factor analysis. British Journal of Psychology, Statistical Section, $1952,5,1-6$.

Ledermann, W. The orthogonal transformation of a factorial matrix into itself. Psychometrika, 1938, 3, $181-187$.

McDonald, R. P. The measurement of factor indeterminacy. Psychometrika, 1974, 39, 203-221.

McDonald, R. P., \& Burr, E. J. A comparison of four methods of constructing factor scores. Psychometrika, $1967,32,381-401$.

Meyer, E. P. On the relationship between ratio of number of variables to number of factors and factorial indeterminacy. Psychometrika, 1973, 38, 375-378.

Mulaik, S. A. The foundations of factor analysis. New York: McGraw-Hill, 1972.

Mulaik, S. A. Comments on 'the measurement of factorial indeterminacy.' Psychometrika, 1976, 4l, $249-262$.

Mulaik, S. A., \& McDonald, R. P. The effect of additional variables on factor indeterminancy in models with a single common factor. Psychometrika, 1978, 43, 177-192.

Piaggio, H. T. H. The general factor in Spearman's theory of intelligence. Nature, 1931, 127, $56-57$. 
Piaggio, H. T. H. Three sets of conditions necessary for the existence of a $g$ that is real and unique except in sign. British Journal of Psychology, 1933, 24, 88-105.

Piaggio, H. T. H. Approximate general and specific factors without indeterminate parts. British Journal of Psychology, 1935, 25, 485-489.

Piaggio, H. T. H., \& Dallas, A. E. M. N. An analysis of recent tests of the two-factor theory. British Journal of Psychology, 1934, 25, 217-220.

Schönemann, P. H. The minimum average correlation between equivalent sets of uncorrelated factors. Psychometrika, 1971, 36, 21-30.

Schönemann, P. H., \& Wang, M. M. Some new results on factor indeterminacy. Psychometrika, 1972, 37, 61-91.

Schönemann, P. H., \& Steiger, J. H. Regression component analysis. British Journal of Mathematical and Statistical Psychology, 1976, 29, 175-189.

Schönemann, P. H., \& Steiger, J. H. On the validity of indeterminate factor scores. Bulletin of the Psychonomic Society, 1978, 12, 287-290.

Spearman, C. General intelligence, objectively determined and measured. American Journal of Psychology. 1904, 15, 201-293.

Spearman, C. Correlation between arrays in a table of correlations. Proceedings of the Royal Society, Series A, $1922,101,94-100$.

Spearman, C. The abilities of man. New York: MacMillan, 1927.

Spearman, C. The uniqueness of ' $g$ '. Journal of Educational Psychology, 1933, 24, 106-108.

Spearman, C. The factor theory and its troubles. IV. Uniqueness of g. Journal of Educational Psychology, 1934, $25,142-153$.

Steiger, J. H., The relationship between external variables and indeterminate factors. Psychometrika, 1979, 44, 93-97.

Thomson, G. H. A hierarchy without a general factor. British Journal of Psychology, 1916, 8, 271-281.

Thomson, G. H. The hierarchy of abilities. British Journal of Psychology, 1919, 9, 337-344.

Thomson, G. H. The meaning of ' $i$ ' in the measurement of ' $g$ ' (general intelligence). Journal of Educational Psychology, 1935, 26, 241-262.

Thurstone, L. L. The vectors of mind. Chicago: University of Chicago Press, 1935.

Thurstone, L. L. Multiple factor analysis. Chicago: University of Chicago Press, 1947.

Williams, J. S. A definition for the common factor model and the elimination of problems of factor score indeterminancy. Psychometrika, 1978, 43, 293-306.

Wilson, E. B. Review of 'The abilities of man, their nature and measurement' by C. Spearman. Science, 1928a, 67, 244-248.

Wilson, E. B. On hierarchical correlation systems. Proceedings of the National Academy of Sciences, 1928b, 14 , 283-291.

Wilson, E. B. Review of 'Crossroads in the mind of man: A study of differentiable mental abilities' by T. L. Kelley. Journal of General Psychology, 1929a, 2, 153-169.

Wilson, E. B. Comments on Professor Spearman's note. Journal of Educational Psychology, 1929b, 20, 217-223.

Wolfle, D. Factor analysis to 1940. Psychometric Monographs, No. 3, Chicago: University of Chicago Press, 1940.

Manuscript received $4 / 21 / 76$

Final version received $3 / 7 / 78$ 